\title{
Anatomical structure of the coracohumeral ligament and its effect on shoulder joint stability
}

\author{
C. Sun, B. Zhong, Z. Pan, D. Du, X. Min \\ Department of Joint Surgery, the Eighty-Ninth Hospital of People's Liberation Army, Weifang, China
}

[Received: 1 August 2016; Accepted: 16 December 2016]

Background: In this study, coracohumeral ligament (CHL) specimens were carefully dissected to observe its length, width, thickness and tension at different positions of the shoulder joint, thereby elucidating its effects on shoulder joint stability.

Materials and methods: Fresh frozen shoulder joints from 40 normal adult cadaveric specimens were dissected to reveal the CHL. With the shoulder joints placed at different positions, the length of the CHL and the width and thickness of the middle part of the ligament were measured. The changes in tension of the CHL were also observed. When the shoulder joint maintained the neutral position, the length of the CHL was $52.23 \pm 1.02 \mathrm{~mm}$ and the width and thickness of the middle part of the ligament were $15.95 \pm 0.59 \mathrm{~mm}$ and $1.46 \pm 0.06 \mathrm{~mm}$, respectively.

Results: When the shoulder joint moved from the neutral position to $90^{\circ}$ external rotation, from the neutral position to $30^{\circ}$ adduction or from the neutral position to $30^{\circ}$ flexion/extension or when the shoulder joint is pulled down with a $5 \mathrm{~kg}$ weight, the CHL was elongated and thinned, maintaining a strained state. When the shoulder joint moved from the neutral position to $90^{\circ}$ internal rotation, from the neutral position to $90^{\circ}$ abduction or from the neutral position to $30^{\circ}$ flexion/ extension, the CHL was shortened and thickened, maintaining a relaxed state. Conclusions: The CHL may limit the external rotation, adduction and downward movement of the shoulder joint and the process from the neutral position to the $30^{\circ}$ flexion/extension, maintaining shoulder joint stability. (Folia Morphol 2017; 76, 4: 720-729)

Key words: coracohumeral ligament, anatomy, measurement, shoulder join, stability

\section{INTRODUCTION}

The glenohumeral $(\mathrm{GH})$ joint is also called the shoulder joint and is the most flexible ball-and-socket joint of the human body [3]. The flexible features of the GH joint depend primarily on two anatomical factors:

1. shallow glenoid and large humeral articular surface (the humeral articular surface is three times larger than the glenoid), with the humeral articular angle approximately $135^{\circ}$ and the glenoid angle only $75^{\circ}$ or so, and

2. relatively weak GH joint ligament [1]. However, these two anatomical factors are vulnerable to GH joint dislocation after injury, and the dislocation rate accounts for approximately $50 \%$ of joint dislocations all over the body [13, 22]. 
Maintaining the GH joint stability mainly relies on the rotator cuff around the joint and the capsular ligament [1]. In 1970, Neer [16] first proposed the term "rotator interval" (RI) to describe the histological structure between supraspinatus tendon and subscapularis tendon, consisting of the coracohumeral ligament (CHL), the superior glenohumeral ligament (SGHL) and a part of the anterior articular capsule, and verified that the RI played a role in maintaining shoulder joint stability in subsequent studies with Foster [17]. Since Neer's study, Neer et al. [18] reported in 1992 that the $\mathrm{CHL}$ played an important limiting role on the external rotation of the shoulder joint; $\mathrm{CHL}$ release or amputation for patients with frozen shoulder joint obstruction during external rotation began clinically, having significant influences on the long-term anterior shoulder stability $[2,11,14]$. In this study, the length, width and thickness of the $\mathrm{CHL}$ at a specific position of the shoulder joint are measured anatomically, and changes in tension are described by observing the contraction state, with a view to illuminate the specific action mechanisms of $\mathrm{CHL}$ on maintaining shoulder joint stability.

\section{MATERIALS AND METHODS}

\section{Materials}

Fresh frozen shoulder joints were taken from 40 normal adult cadaveric specimens, consisting of 27 males and 13 females with the median age of 47.5 (19-57) years and 18 left shoulders and 22 right shoulders with intact skin and free of deformities, traumas and surgical alterations. This study was conducted in accordance with the declaration of Helsinki. This study was conducted with approval from the Ethics Committee of the Eighty-Ninth Hospital of People's Liberation Army. Written informed consent was obtained from all participants.

\section{Anatomical observations of coracohumeral ligament}

Conventional surgical instruments (Beijing Medical Instruments Factory, Beijing, China) were used to dislodge skin and subcutaneous tissues from the specimens (Fig. 1), with the deltoid and pectoralis major amputated from the starting point and opened to the ending point. Coracoclavicular ligament and capsula articularis acromioclavicularis were amputated in turn and the clavicle was dislodged (Figs. 2, 3). Whether the ending point of the pectoralis major

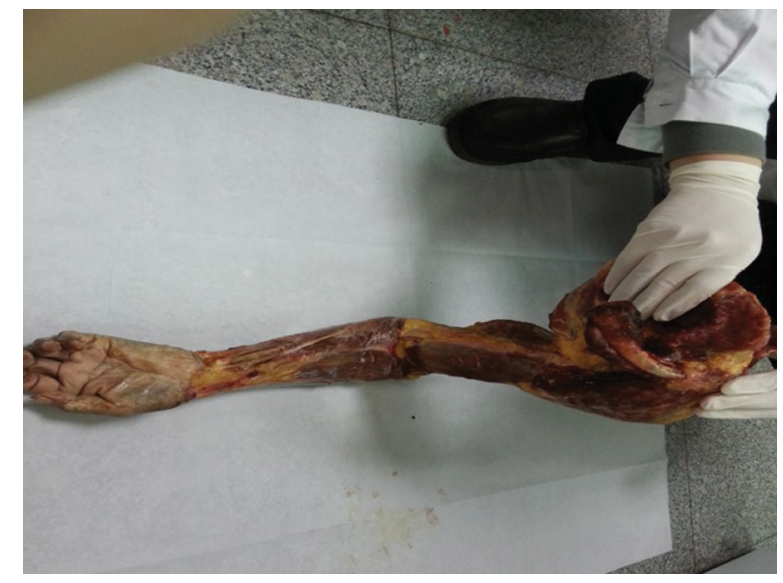

Figure 1. Specimens with skin and subcutaneous tissues dislodged.

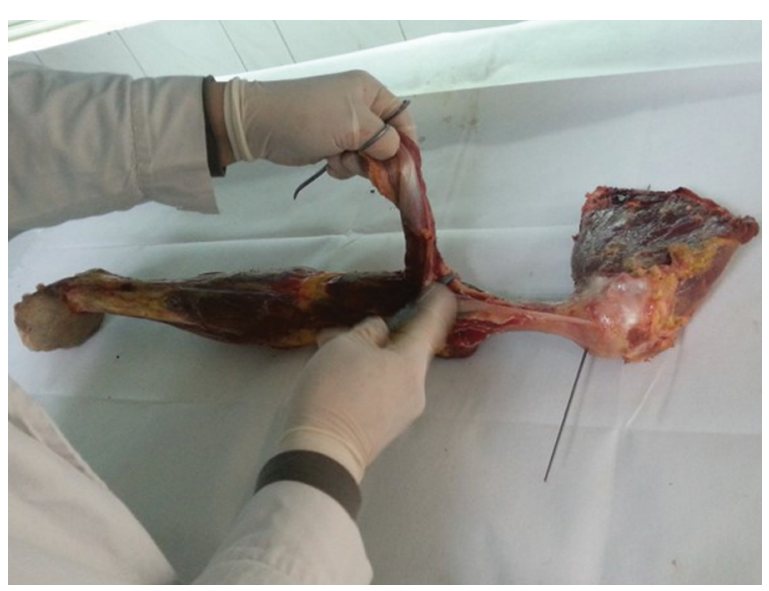

Figure 2. Removed deltoid, pectoralis major, coracoclavicular ligament and capsula articularis acromioclavicularis.

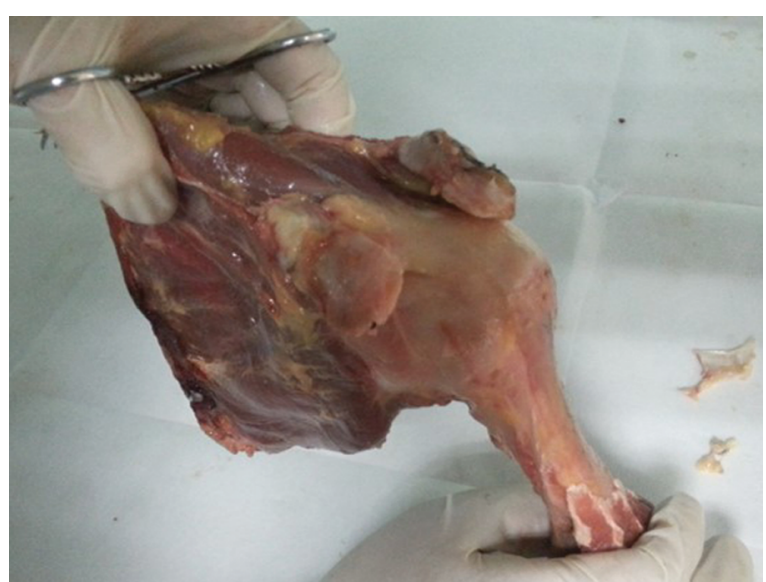

Figure 3. Removed deltoid, pectoralis major, coracoclavicular ligament and capsula articularis acromioclavicularis and dislodged clavicle. 


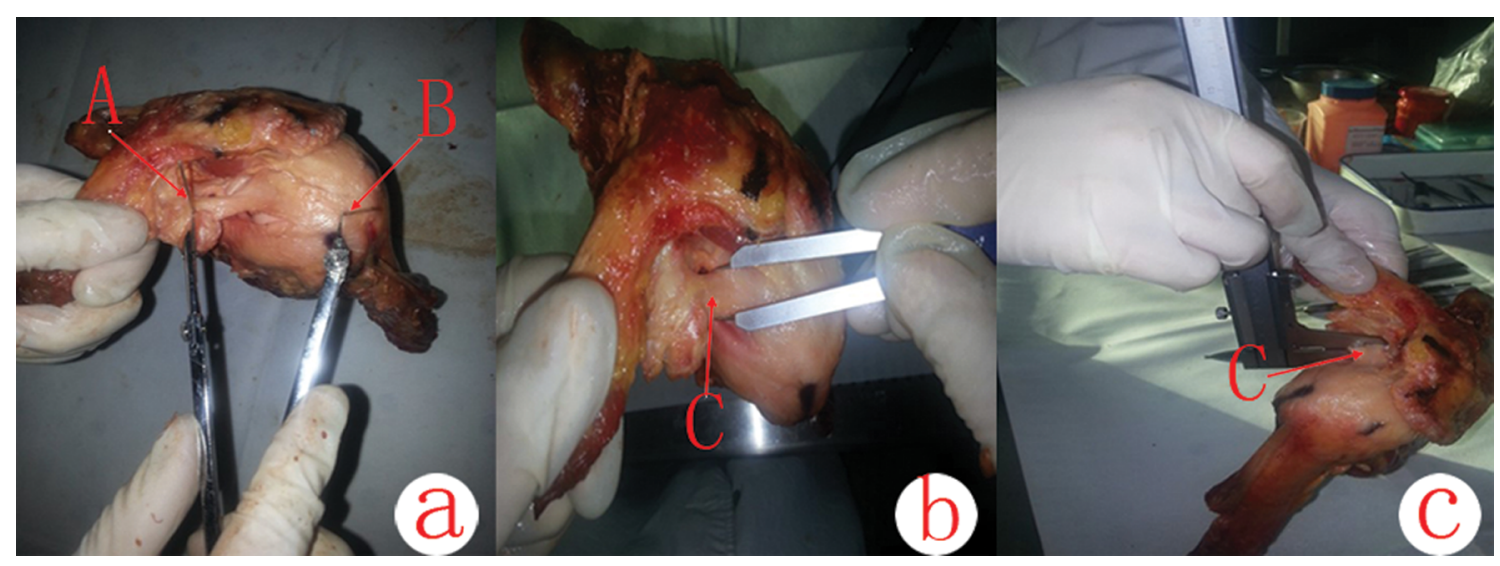

Figure 4. Measurement data in the normal neutral standing position; a. Measurement of the length of coracohumeral ligament (CHL): two $1.0 \mathrm{~mm}$ Kirschners needles were perpendicularly stroke into the initiation points of $\mathrm{CHL}$ (marked as point $\mathrm{A}$ and $\mathrm{B}$ ), the distance between point $\mathrm{A}$ and $\mathrm{B}$ was then marked using one compass, followed by precision measurement using one sliding calliper; $\mathbf{b}$. Measurement of the mid-length of CHL: the mid-point of CHL was marked as point $\mathrm{C}$, the width of which was measured using marked using one compass and precisely measured using one sliding calliper; c. Measurement of the mid-thickness of CHL: the thickness of CHL was directly measured using one sliding calliper at point $\mathrm{C}$.

extended from the tip of the coracoid and migrated to the capsula articularis humeri was observed. In case of any variation in the pectoralis minor, the pectoralis minor tendon might cross the $\mathrm{CHL}$ and intermingle with the moving part of the coracoid, and insignificant anatomical differences between them might be observed, such that the moving part was included in the present study as the CHL [26]. The pectoralis major, the coracobrachialis and the short head of the biceps femoris were amputated from the edge of the coracoid. The RI was exposed after all fatty tissues underneath the coracoacromial ligament and the subacromial bursa were dislodged, and the RI and its adjacent structures were observed [6]. The ending point of the coracoid below the CHL was revealed after the coracoacromial ligament was dislodged, the free edge of the $\mathrm{CHL}$ was dissected along the ending point of the coracoid until the ending point of the humerus was reached and the undersurface was cut from the articular capsule along the edge [26].

\section{Data measurements}

\section{of the coracohumeral ligament}

Measuring the compliant specimens:

3. When the shoulder joints maintained the neutral position, the length of the $\mathrm{CHL}$ and the width and thickness of the middle part of the ligament were measured with vernier callipers (precision = $0.02 \mathrm{~mm}$; Mitutoyo-Instruments, Fujian, China) to obtain three sets of data, shown in Figure 4.
The SPSS19.0 software (IBM Corporation, USA) was employed for the statistical analysis, with the results expressed as $x \pm s$.

4. The normal range of shoulder motion is internal rotation about $70^{\circ}-90^{\circ}$; external rotation about $60^{\circ}-90^{\circ}$; adduction about $20^{\circ}-40^{\circ}$; abduction about $160^{\circ}-180^{\circ}$; anteflexion about $150^{\circ}-170^{\circ}$; retroflexion about $40^{\circ}-45^{\circ}$, we chose this normal range and trial tests for measurements.

a) The shoulder joint specimens were placed in the internal rotation angles of $30^{\circ}, 60^{\circ}$ and $90^{\circ}$ and external rotation angles of $30^{\circ}, 60^{\circ}$ and $90^{\circ}$ using universal bevel protractors (Xuzhou Granville Electrical Co., Ltd., Xuzhou, China), shown in Figure 5. Two 2.0-mm Kirschner needles (Kirschner needle A and B) were fixed along the two measurement edges of one universal bevel protractor as the reference objects for the measurement. When the specimen's shoulder joint was in the normal neutral position, one $2.0 \mathrm{~mm}$ Kirschners needle (Kirschner needle C) was stroke into the midpoint of humeral greater tubercle and perpendicular to the humerus as the reference object for the rotation angle of the shoulder joint. The specimen's shoulder joint was then placed on the level of the dissecting table, and the universal bevel protractor was placed above the shoulder so that the rotation centre was positioned at 


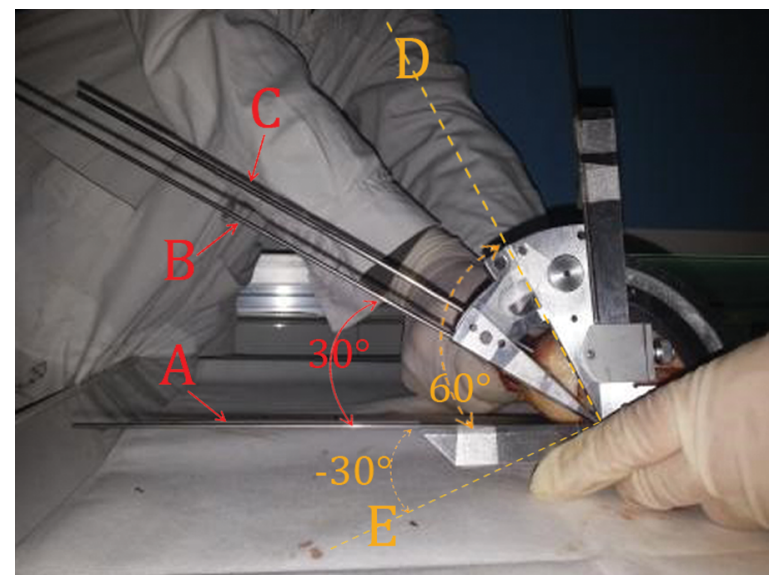

Figure 5. Positioning and data measurement of internal and external rotation. Description in the text.

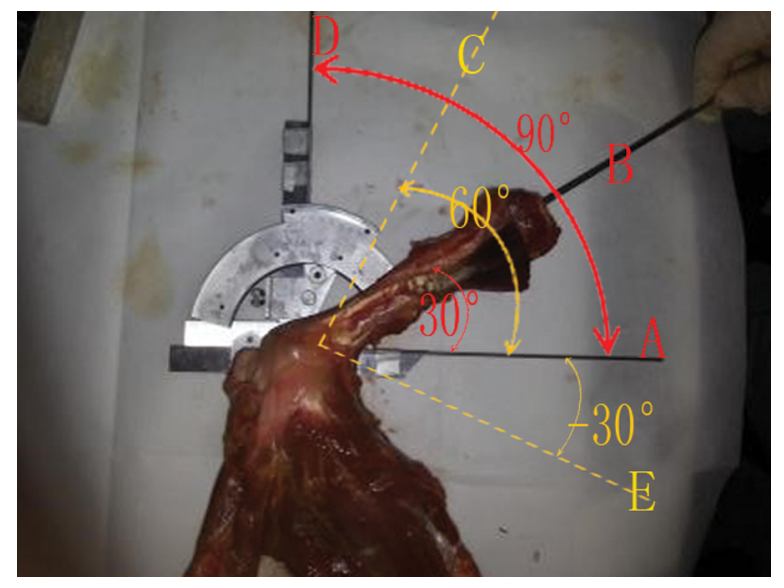

Figure 6. Positioning and data measurement of internal contract and external extension. Description in the text.

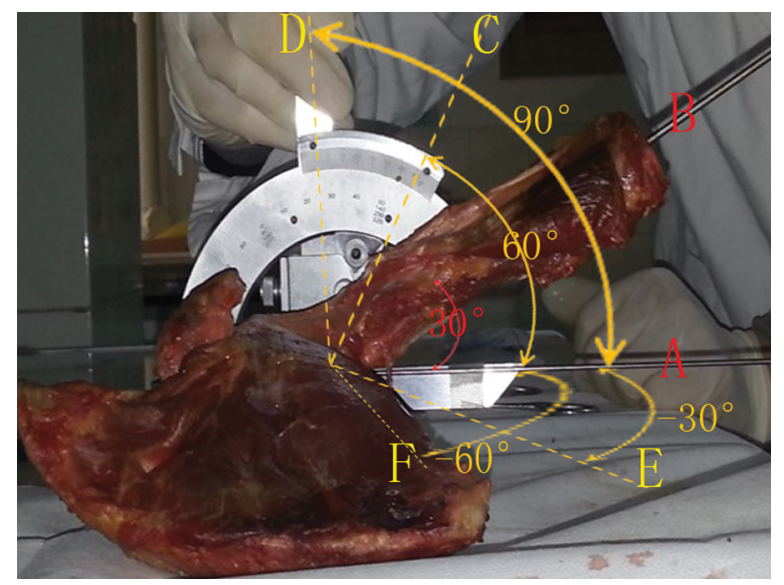

Figure 7. Positioning and data measurement of procurvation and backward extension. Description in the text. the rotation centre of humeral head; rotated the shoulder joint, and gradually opened the universal bevel protractor with the shoulder joint while always maintained the Kirschner needles $B$ and $C$ parallel to each other; read the scale data and positioned the points. When the Kirschner needle $B$ rotated to the position shown in the Figure 5, the shoulder joint inward-rotated $30^{\circ}$; when the Kirschner needle $B$ rotated to the line $D$, the shoulder joint inward-rotated $60^{\circ}$; when the Kirschner needle $B$ rotated to the line $D$, the shoulder joint externally rotated $30^{\circ}$. In addition, the three sets of data were measured with vernier callipers, recorded and analysed.

b) In the same manner, the shoulder joint specimens were placed and measured in the adduction angle of $30^{\circ}$ and abduction angles of $30^{\circ}, 60^{\circ}$ and $90^{\circ}$ (Fig. 6). The Kirschner needle $A$ was fixed at the humeral long axis of shoulder specimen in normal neutral standing position, and then the shoulder specimen and the Kirschner needle B were rotated so as to maintain the humeral long axis continuously coincide with the Kirschner needle $B$; the data and position were then read using the universal bevel protractor. When the Kirschner needle $B$ was in the position shown in the Figure 6 , the shoulder joint externally extended $30^{\circ}$; when the Kirschner needle $B$ was rotated to the line $C$, the shoulder joint externally extended $60^{\circ}$; when the Kirschner needle $B$ was rotated to the line $D$, the shoulder joint externally extended $90^{\circ}$; when the Kirschner needle B was rotated to the line $E$, the shoulder joint internally contracted $30^{\circ}$.

c) Flexion angles of $30^{\circ}, 60^{\circ}$ and $90^{\circ}$ and extension angles of $30^{\circ}$ and $60^{\circ}$ (Fig. 7). The shoulder joint was firstly fixed horizontally, and the universal bevel protractor was perpendicularly placed and closely attached to the lateral side of the shoulder to make the Kirschner needle A being kept in the horizontal place. The shoulder specimen and the Kirschner needle $B$ were then rotated so as to maintain the humeral long axis continuously coincide with the Kirschner needle $B$; the data and position were then read using the universal bevel protractor. When the Kirschner needle $B$ was in the 
Table 1. Coracohumeral ligament (CHL) measurements and analyses between male and female or left and right groups

\begin{tabular}{lccc}
\hline & Length & Width & Thickness \\
\hline Male & $50.91 \pm 1.05$ & $15.71 \pm 0.49$ & $1.49 \pm 0.05$ \\
Female & $50.62 \pm 1.07$ & $15.97 \pm 0.61$ & $1.39 \pm 0.09$ \\
$\mathrm{p}$ & $<0.05$ & $<0.05$ & $<0.05$ \\
Left & $50.89 \pm 1.06$ & $16.01 \pm 0.47$ & $1.42 \pm 0.07$ \\
Right & $50.77 \pm 1.02$ & $15.77 \pm 0.43$ & $1.41 \pm 0.06$ \\
$\mathrm{p}$ & $<0.05$ & $<0.05$ & $<0.05$ \\
\hline
\end{tabular}

CHL measurements and analyses between left and right groups

Table 2. Coracohumeral ligament (CHL) measurements and analyses of the shoulder joint specimens in internal/external rotation

\begin{tabular}{lccc}
\hline Positions of the shoulder joint specimens & \multicolumn{3}{c}{ CHL measurements and analyses } \\
\cline { 2 - 4 } & Length [mm] & Width in the middle [mm] & Thickness in the middle [mm] \\
\hline Internal rotation $90^{\circ}$ & $21.07 \pm 0.56$ & $16.20 \pm 0.47$ & $1.59 \pm 0.07$ \\
Internal rotation $60^{\circ}$ & $31.92 \pm 0.46$ & $16.12 \pm 0.57$ & $1.51 \pm 0.06$ \\
Internal rotation $30^{\circ}$ & $34.76 \pm 0.55$ & $16.07 \pm 0.63$ & $1.49 \pm 0.04$ \\
Neutral position & $50.79 \pm 1.02$ & $15.95 \pm 0.59$ & $1.46 \pm 0.06$ \\
External rotation $30^{\circ}$ & $58.41 \pm 0.44$ & $14.32 \pm 0.56$ & $1.43 \pm 0.05$ \\
External rotation $60^{\circ}$ & $70.69 \pm 0.37$ & $14.29 \pm 0.44$ & $1.40 \pm 0.07$ \\
External rotation $90^{\circ}$ & $80.77 \pm 0.63$ & $14.26 \pm 0.62$ & $1.37 \pm 0.09$ \\
\hline
\end{tabular}

position shown in the Figure 7, the shoulder joint was $30^{\circ}$ of antexion; when the Kirschner needle $B$ was rotated to the line $C$, the shoulder joint was $60^{\circ}$ of antexion; when the Kirschner needle $B$ was rotated to the line $D$, the shoulder joint was $90^{\circ}$ of antexion; when the Kirschner needle $B$ was rotated to the line $E$, the shoulder joint backward extended $30^{\circ}$; when the Kirschner needle B was rotated to the line $F$, the shoulder joint backward extended $60^{\circ}$. The three sets of data were measured, recorded and analysed. Finally, the shoulder joints were placed in the neutral position and pulled down with a $5 \mathrm{~kg}$ weight, and the three sets of data were measured, recorded and analysed.

\section{Tension observations of the coracohumeral ligament}

The tension of the $\mathrm{CHL}$ was observed when the shoulder joint specimens were under each of the following conditions: neutral position, internal rotation, external rotation, flexion, extension, adduction, abduction and being pulled down with a $5 \mathrm{~kg}$ weight.

\section{Coracohumeral ligament measurements}

The $\mathrm{CHL}$ specimens complying with the inclusion criteria were measured, and when the shoulder joint specimens were in the neutral position, the length of the CHL was $50.79 \pm 1.02 \mathrm{~mm}$ the width and thickness of the middle part of the ligament were $15.95 \pm 0.59 \mathrm{~mm}$ and $1.46 \pm 0.06 \mathrm{~mm}$, respectively. Moreover, there was no statistical difference between male and female or left and right groups $(p<0.05)$ (Table 1). When the shoulder joint specimens were at the internal rotation angles of $30^{\circ}$, $60^{\circ}$ and $90^{\circ}$ and external rotation angles of $30^{\circ}, 60^{\circ}$ and $90^{\circ}$, the measured length of the $\mathrm{CHL}$ and the width and thickness of the middle part of the ligament were expressed as $x \pm s$, as shown in Table 2 . With the adduction angle of $30^{\circ}$ and the abduction angles of $30^{\circ}, 60^{\circ}$ and $90^{\circ}$, the three sets of data are shown in Table 3. With the flexion angles of $30^{\circ}, 60^{\circ}$ and $90^{\circ}$ and the extension angles of $30^{\circ}$ and $60^{\circ}$, the three sets of data are shown in Table 4. When the shoulder joint specimens were pulled down with a $5 \mathrm{~kg}$ weight, the three sets of data are shown in Table 5 . 
Table 3. Coracohumeral ligament (CHL) measurements and analyses of the shoulder joint specimens in adduction/abduction

\begin{tabular}{lccc}
\hline Positions of the shoulder joint specimens & \multicolumn{3}{c}{ CHL measurements and analyses } \\
\cline { 2 - 4 } & Length [mm] & Width in the middle [mm] & Thickness in the middle [mm] \\
\hline Adduction $30^{\circ}$ & $53.31 \pm 0.87$ & $15.87 \pm 0.42$ & $1.44 \pm 0.04$ \\
Neutral position & $50.79 \pm 1.02$ & $15.95 \pm 0.59$ & $1.46 \pm 0.06$ \\
Abduction $30^{\circ}$ & $44.17 \pm 0.77$ & $16.09 \pm 0.66$ & $1.50 \pm 0.07$ \\
Abduction $60^{\circ}$ & $43.47 \pm 0.64$ & $16.12 \pm 0.75$ & $1.51 \pm 0.11$ \\
Abduction $90^{\circ}$ & $39.81 \pm 0.69$ & $16.21 \pm 0.45$ & $1.52 \pm 0.05$ \\
\hline
\end{tabular}

Table 4. Coracohumeral ligament (CHL) measurements and analyses of the shoulder joint specimens in flexion/extension

\begin{tabular}{lccc}
\hline Positions of the shoulder joint specimens & \multicolumn{3}{c}{ CHL measurements and analyses } \\
\cline { 2 - 4 } & Length [mm] & Width in the middle [mm] & Thickness in the middle [mm] \\
\hline Flexion $90^{\circ}$ & $43.71 \pm 0.61$ & $15.78 \pm 0.56$ & $1.50 \pm 0.06$ \\
Flexion $60^{\circ}$ & $46.41 \pm 0.79$ & $15.81 \pm 0.76$ & $1.48 \pm 0.05$ \\
Flexion $30^{\circ}$ & $54.72 \pm 0.80$ & $15.91 \pm 0.47$ & $1.45 \pm 0.07$ \\
Neutral position & $50.79 \pm 1.02$ & $15.95 \pm 0.59$ & $1.46 \pm 0.06$ \\
Extension $30^{\circ}$ & $52.76 \pm 1.65$ & $15.93 \pm 0.69$ & $1.45 \pm 0.09$ \\
Extension $60^{\circ}$ & $47.20 \pm 0.66$ & $15.79 \pm 0.46$ & $1.48 \pm 0.03$ \\
\hline
\end{tabular}

Table 5. Coracohumeral ligament (CHL) measurements and analyses of the shoulder joint specimens being pulled down with a $5 \mathrm{~kg}$ weight

\begin{tabular}{lccc}
\hline Positions of the shoulder joint specimens & \multicolumn{3}{c}{ CHL measurements and analyses } \\
\cline { 2 - 4 } & Length $[\mathrm{mm}]$ & Width in the middle [mm] & Thickness in the middle [mm] \\
\hline Neutral position & $50.79 \pm 1.02$ & $15.95 \pm 0.59$ & $1.46 \pm 0.06$ \\
Being pulled down with a 5 kg weight & $69.53 \pm 0.42$ & $14.43 \pm 0.37$ & $1.41 \pm 0.05$ \\
\hline
\end{tabular}

\section{Changes in the tension}

\section{of the coracohumeral ligament}

The CHL did not have any lacklustre surface or any obvious tension on the rigidly connected bones like the coracoacromial ligament. The tension of the $\mathrm{CHL}$ in the neutral position was taken as a reference basis. The $\mathrm{CHL}$ in $\pm 30^{\circ}$ external rotation/flexion/extension angle, from the neutral position to $30^{\circ}$ adduction or when the shoulder joint being pulled down with a $5 \mathrm{~kg}$ weight was under strain. By contrast, the $\mathrm{CHL}$ in internal rotation, abduction or continual flexion/ /extension from $\pm 30^{\circ}$ was in a relaxed state.

\section{DISCUSSION}

Currently studies on the coracohumeral ligament

Since Neer [16] first proposed to describe the portion of the rotator cuff between supraspinatus tendon and subscapularis tendon with the term "rotator interval" in 1970, many scholars have conducted a series of dissected anatomical and biomechanical studies on the RI and its composition [12, 21, 25]. In 1980, Neer and Foster [17] verified the effects of the RI on anterior shoulder stability. In 1987, Nobuhara and Ikeda [19] first reported and classified patients with injuries to the RI. After years of development, the RI has become a recognised independent anatomical structure maintaining shoulder joint stability in normal activities, has been the focus of considerable attention from scholars and has been widely considered the main structure bearing the RI functions [8].

Early in 1959, Basmajian and Bazant [4] reported that the anterior articular capsule and its associated ligaments countered a shift downward during shoulder abduction. Since then, the normal physiological function of the $\mathrm{CHL}$ has been reported in many studies. By selectively amputating the $\mathrm{CHL}$ and $\mathrm{SGHL}$, Ovesen and Nielsen [20] observed that the $\mathrm{CHL}$ played 
an important role in maintaining the downward shoulder joint stability from the neutral position. Similarly, by analysing two kinds of ligament stretching resistances, Wellmann et al. [24] determined that the strength and ultimate load capacity of the $\mathrm{CHL}$ were greater than that of the SGHL. Therefore, they concluded that the $\mathrm{CHL}$ played a major role in maintaining shoulder joint stability at the RI [15]. However, the mechanism of action of the $\mathrm{CHL}$ in maintaining shoulder joint stability has not been clarified. In 1992, on the basis of their cadaver experiments on the RI functions, Harryman et al. [9] concluded that the RI could limit any excessive flexion, extension, adduction and/or external rotation of the shoulder joint and prevent the shoulder joint from any fall in adduction; however, the functions of structures and/or components inside the RI were not explained in detail. Therefore, in this study, the specific roles of the $\mathrm{CHL}$ on shoulder joint stability were separately explored based on the relevant measurements of functional anatomy.

\section{Analysis of the measured data of the coracohumeral ligament in the present study}

In the present study, 23 of the 40 anatomical specimens revealed fibrous tissues connecting the $\mathrm{CHL}$ and SGHL (i.e., the so-called CHL/SGHL complex) $[5,23]$, and the remaining 17 did not show any obvious distinction between the SGHL and the articular capsule, which indirectly indicated that the $\mathrm{CHL}$ was the thickened portion of the articular capsule that strengthened the upper part of the articular capsule, limited the external humeral rotation and avoided the upward humeral dislocation [23].

When the shoulder joint was in internal or external rotation, the correlation between the motion angle of the shoulder joint and the measured data (the length of the CHL and the width and thickness of the middle part of the ligament) was analysed by grouping and curve fitting. The resultant correlation coefficient was $0<R<1$, indicating that a certain relationship exists among the motion angle of the shoulder joint, the length of the $\mathrm{CHL}$ and the width and thickness of the middle part of the ligament. Figure 8 shows that the $\mathrm{CHL}$ in the internal rotation of the shoulder joint was gradually shortened, widened and thickened. Moreover, during the $90^{\circ}$ external rotation of the shoulder joint from the neutral position, the $\mathrm{CHL}$ was elongated, narrowed and thinned. These findings indicate that the $\mathrm{CHL}$ could limit the external rotation of the shoulder joint.
When the shoulder joint was in the adduction/ abduction process, the data processing method used was the same as that previously described. The resultant correlation coefficient was $0<\mathrm{R}<1$, indicating that the $\mathrm{CHL}$ in $30^{\circ}$ adduction of the shoulder joint from the neutral position was elongated, narrowed and thinned and the $\mathrm{CHL}$ in the process of shoulder joint abduction gradually shortened, widened and thickened (Fig. 9). Thus, the CHL could limit the abduction of the shoulder joint.

When the shoulder joint was in the flexion/extension process, the data processing method used was the same as that previously described. The resultant correlation coefficient was $0<\mathrm{R}<1$, indicating that the $\mathrm{CHL}$ in $30^{\circ}$ flexion of the shoulder joint from the neutral position was elongated, narrowed and thinned and the $\mathrm{CHL}$ in continual flexion gradually shortened, widened and thickened. Moreover, the $\mathrm{CHL}$ in $30^{\circ}$ extension of the shoulder joint from the neutral position was elongated, narrowed and thinned and the $\mathrm{CHL}$ in continual extension gradually shortened, widened and thickened (Fig. 10). These findings indicated that the $\mathrm{CHL}$ had certain limitations within the $\pm 30^{\circ}$ flexion/extension of the $\mathrm{GH}$ joint; thus, the $\mathrm{CHL}$ could maintain the initial $\mathrm{GH}$ joint stability.

When the shoulder joint specimens were being pulled down with a $5 \mathrm{~kg}$ weight, the three sets of data were measured and compared with those in the neutral position (Table 5). The results show that, when the shoulder joint was being pulled down with a $5 \mathrm{~kg}$ weight, the $\mathrm{CHL}$ was elongated, narrowed and thinned. This finding indicated that the $\mathrm{CHL}$ could limit the downward movement of the shoulder joint; thus, the $\mathrm{CHL}$ could maintain the downward $\mathrm{GH}$ joint stability. Therefore, several scholars have proposed that, in the event that the shoulder joint becomes unstable in a certain direction, the RI could be closed to increase the downward shoulder joint stability $[7,10]$.

In summary, the CHL has certain limitations on the external rotation, adduction and downward movement of the shoulder joint, maintaining a certain tension within the scope of $\pm 30^{\circ}$ flexion/extension of the $\mathrm{GH}$ joint. Thus, the $\mathrm{CHL}$ is an important structure that maintains the shoulder joint stability.

\section{Deficiencies and limitations of the present study}

The present experiment has a small sample size, and owing to the incomplete information source of 


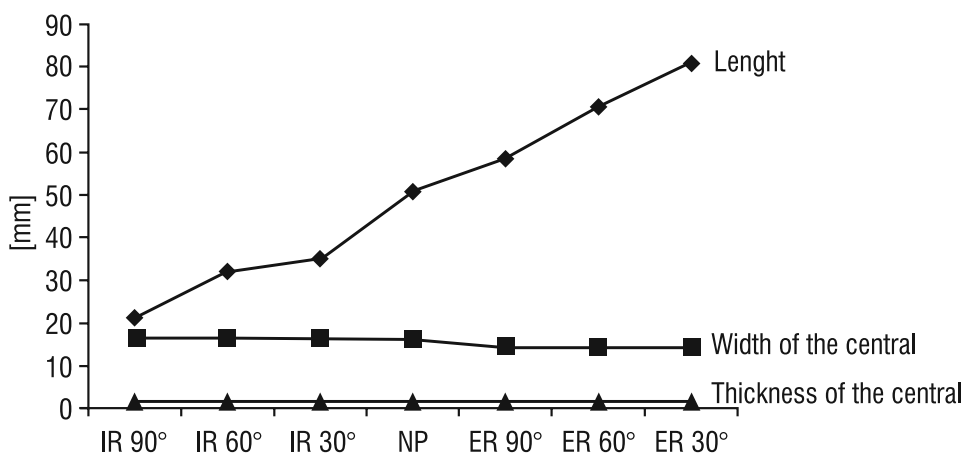

Figure 8. Variations in the measured coracohumeral ligament data during internal/external rotation of the shoulder joint; ER — external rotation; $\mathrm{IR}$ - internal rotation; NP - neutral position.

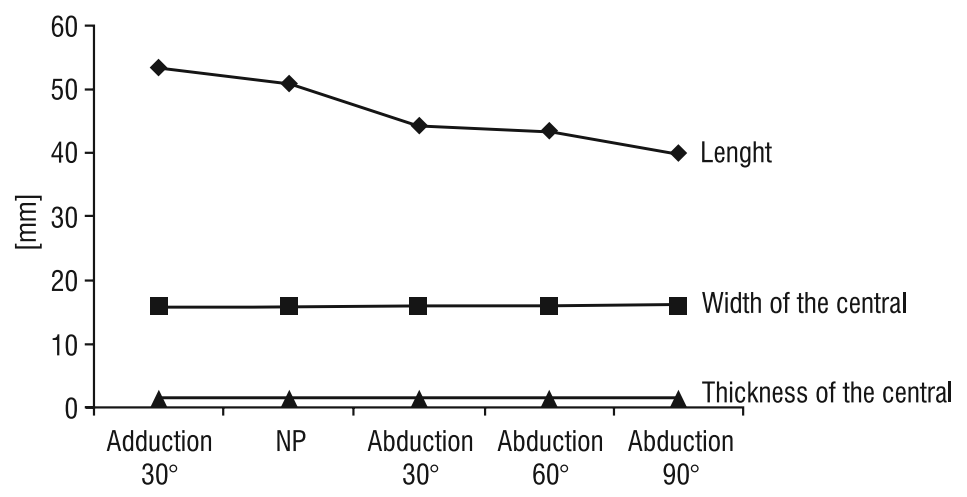

Figure 9. Variations in the measured coracohumeral ligament data during adduction/abduction of the shoulder joint; NP — neutral position.

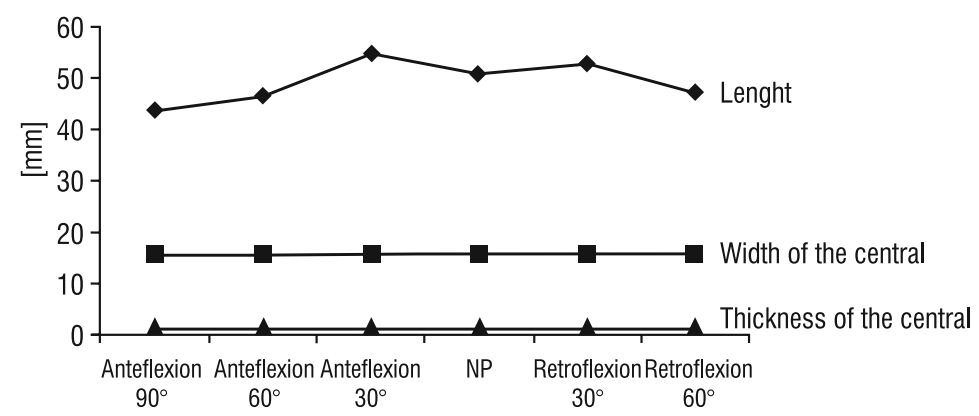

Figure 10. Variations in the measured coracohumeral ligament data during flexion/extension of the shoulder joint; NP — neutral position.

the specimens, specimen information errors, such as height, weight, age and residence of the donors, may lead to incorrect results or different mean values from the population mean.

During the movements of the shoulder joint, the caput humeri may also slide inside the glenoid, thus changing the trajectory of the caput humeri and leading to errors in measuring the $\mathrm{CHL}$ length at different angles.

When measuring the $\mathrm{CHL}$ data at different angles, the angular position is simple and the different planes where the humerus moves are incompletely parallel to the test bench, thus resulting in measurement errors. 


\section{CONCLUSIONS}

The present study is a retrospective study based on the assumption that the $\mathrm{CHL}$ could limit the external rotation and adduction of the shoulder joint and maintain the shoulder joint stability in the movements previously described. However, whether the $\mathrm{CHL}$ could limit the downward movement of the shoulder joint is not clarified. The retrospective analysis of 40 shoulder joint specimens showed that the CHL could not only limit the external rotation, adduction and downward movement of the shoulder joint but also maintain a certain tension within the $\pm 30^{\circ}$ flexion/extension of the $\mathrm{GH}$ joint. The results are in line with the expectations and hypotheses, and the present study can not only verify that the $\mathrm{CHL}$ maintains the shoulder joint stability but also argue that the $\mathrm{CHL}$ changes with the $\mathrm{GH}$ joint movement in different directions. This study provides a theoretical basis for clinically determining the specific mechanism of injuries due to $\mathrm{GH}$ joint instability.

\section{Acknowledgements}

This study was conducted in accordance with the declaration of Helsinki. This study was conducted with approval from the Ethics Committee of the Eighty-Ninth Hospital of People's Liberation Army. Written informed consent was obtained from all participants.

\section{REFERENCES}

1. Antunes JP, Mendes A, Prado MH, et al. Arthroscopic Bankart repair for recurrent shoulder instability: A retrospective study of 86 cases. J Orthop. 2016; 13(2): 95-99, doi: 10.1016/j. jor.2016.02.002, indexed in Pubmed: 27053840.

2. Arce G. Primary Frozen Shoulder Syndrome: Arthroscopic Capsular Release. Arthrosc Tech. 2015; 4(6): e717-e720, doi: 10.1016/j.eats.2015.06.004, indexed in Pubmed: 26870652.

3. Bankart AS. Treatment of congenital dislocation of the hip. Br Med J. 1913; 1(2733): 1044-1046.1, indexed in Pubmed: 20766638.

4. Basmajian JV, Bazant FJ. Factors preventing downward dislocation of the adducted shoulder joint. J Bone Joint Surg Am. 1959; 41(7): 1182-1186, doi: 10.2106/00004623195941070-00002.

5. Bennett WF. Subscapularis, medial, and lateral head coracohumeral ligament insertion anatomy. Arthroscopic appearance and incidence of "hidden" rotator interval lesions. Arthroscopy. 2001; 17(2): 173-180, indexed in Pubmed: 11172247.

6. Daly CA, Hutton WC, Jarrett CD. Biomechanical effects of rotator interval closure in shoulder arthroplasty. J Shoulder Elbow Surg. 2016; 25(7): 1094-1099, doi: 10.1016/j. jse.2015.12.003, indexed in Pubmed: 26897316.
7. Frank RM, Taylor D, Verma NN, et al. The rotator interval of the shoulder: implications in the treatment of shoulder instability. Orthop J Sports Med. 2015; 3(12): 2325967115621494, doi: 10.1177/2325967115621494, indexed in Pubmed: 26779554.

8. Haghpanah B, Walley KC, Hingsammer A, et al. The effect of the rotator interval on glenohumeral kinematics during abduction. BMC Musculoskelet Disord. 2016; 17: 46, doi: 10.1186/s12891-016-0898-x, indexed in Pubmed: 26818612.

9. Harryman DT, Sidles JA, Harris SL, et al. The role of the rotator interval capsule in passive motion and stability of the shoulder. J Bone Joint Surg Am. 1992; 74(1): 53-66, doi: 10.2106/00004623-199274010-00008.

10. Itoigawa Y, Itoi E. Anatomy of the capsulolabral complex and rotator interval related to glenohumeral instability. Knee Surg Sports Traumatol Arthrosc. 2016; 24(2): 343-349, doi: 10.1007/s00167-015-3892-1, indexed in Pubmed: 26704796.

11. Kraal T, Visser $C$, Sierevelt $I$, et al. How to treat a frozen shoulder? A survey among shoulder specialists in the Netherlands and Belgium. Acta Orthop Belg. 2016; 82(1): 78-84, indexed in Pubmed: 26984658.

12. Krych AJ, Shindle MK, Baran S, et al. Isolated arthroscopic rotator interval closure for shoulder instability. Arthrosc Tech. 2014; 3(1): e35-e38, doi: 10.1016/j. eats.2013.08.010, indexed in Pubmed: 24749020.

13. Levy DM, Cole BJ, Bach BR. History of surgical intervention of anterior shoulder instability. J Shoulder Elbow Surg. 2016; 25(6): e139-e150, doi: 10.1016/j.jse.2016.01.019, indexed in Pubmed: 27066962.

14. Li Jq, Tang KI, Wang J, et al. MRI findings for frozen shoulder evaluation: is the thickness of the coracohumeral ligament a valuable diagnostic tool? PLoS One. 2011; 6(12): e28704, doi: 10.1371/journal.pone.0028704, indexed in Pubmed: 22163326.

15. Muto $T$, Ninomiya $H$, Inui $H$, et al. Rotator interval lesion and damaged subscapularis tendon repair in a high school baseball player. Case Rep Orthop. 2015; 2015: 890721, doi: 10.1155/2015/890721, indexed in Pubmed: 26618017.

16. Neer CS. Displaced proximal humeral fractures. I. Classification and evaluation. J Bone Joint Surg Am. 1970; 52(6): 1077-1089, indexed in Pubmed:5455339.

17. Neer CS, Foster CR. Inferior capsular shift for involuntary inferior and multidirectional instability of the shoulder. A preliminary report. J Bone Joint Surg Am. 1980; 62(6): 897-908, indexed in Pubmed: 7430177.

18. Neer CS, Satterlee CC, Dalsey RM, et al. The anatomy and potential effects of contracture of the coracohumeral ligament. Clin Orthop Relat Res. 1992(280): 182-185, indexed in Pubmed: 1611740.

19. Nobuhara K, Ikeda H. Rotator interval lesion. Clin Orthop Relat Res. 1987(223): 44-50, indexed in Pubmed: 3652591.

20. Ovesen J, Nielsen S. Posterior instability of the shoulder. A cadaver study. Acta Orthop Scand. 1986; 57(5): 436439, indexed in Pubmed: 3811890.

21. Ponce BA, Rosenzweig SD, Sheppard ED, et al. The effect of arthroscopic rotator interval closure on glenohumeral 
volume. Am J Orthop (Belle Mead NJ). 2015; 44(6): E190-E194, indexed in Pubmed: 26047004.

22. Robinson $\mathrm{CM}$, Howes J, Murdoch $\mathrm{H}$, et al. Functional outcome and risk of recurrent instability after primary traumatic anterior shoulder dislocation in young patients. J Bone Joint Surg Am. 2006; 88(11): 2326-2336, doi: 10.2106/JBJS.E.01327, indexed in Pubmed: 17079387.

23. Visonà $E$, Cerciello $S$, Godenèche $A$, et al. The "comma sign": an anatomical investigation (dissection of the rotator interval in 14 cadaveric shoulders). Surg Radiol Anat. 2015; 37(7): 793-798, doi: 10.1007/s00276-015-1420-0, indexed in Pubmed: 25595552.
24. Wellmann M, Blasig $H$, Bobrowitsch $E$, et al. The biomechanical effect of specific labral and capsular lesions on posterior shoulder instability. Arch Orthop Trauma Surg. 2011; 131(3): 421-427, doi: 10.1007/s00402-010-1232-y, indexed in Pubmed: 21165630.

25. Woertler K. Rotator interval. Semin Musculoskelet Radiol. 2015; 19(3): 243-253, doi: 10.1055/s-0035-1549318, indexed in Pubmed: 26021585.

26. Yang Hf, Tang Kl, Chen $\mathrm{W}$, et al. An anatomic and histologic study of the coracohumeral ligament. J Shoulder Elbow Surg. 2009; 18(2): 305-310, doi: 10.1016/j. jse.2008.07.012, indexed in Pubmed: 19095467. 